\title{
Comparing Conditional Variance Models: Theory and Empirical Evidence
}

\author{
Paolo Girardello \\ University of Verona, Italy \\ Orietta Nicolis \\ University of Bergamo, Italy \\ Giovanni Tondini \\ University of Verona, Italy
}

\begin{abstract}
The aim of this paper is to identify whether the GARCH or the SV based models provide the best goodness of fit to financial time-series data. To investigate the issue, three different formulations for each type (i.e., the standard model, the fat-tailed model, and the asymmetric model) are examined. The models are first compared on theoretical grounds, then estimated using the daily returns from four market indices, and finally subjected to some diagnostic tests. The results demonstrate that for the standard formulation, the SV model fits data better than the GARCH model, while the fat-tailed and the asymmetric models roughly equivalent in describing the key features of returns. The results provide a preliminary analysis for selecting the best model with which to forecast the volatility of financial returns (JEL G0,G1).
\end{abstract}

Keywords: GARCH models, stochastic volatility models, QML estimation, financial time series.

\section{Introduction}

In order to describe the stylized facts which typically characterize the time series of financial returns, such as time-changing variance, clustering, persistence, leverage effect, and strong autocorrelations in the squared returns, two different classes of parametric models, known 
as conditional variance models, are generally applied and studied in the literature: the generalized autoregressive conditionally heteroskedastic (GARCH) models (Engle [1982], Bollerslev [1986]) and the stochastic variance or stochastic volatility (SV) models (Taylor [1986]).

Since Engle's seminal paper (1982) proposing an ARCH model on the basis of the fundamental distinction between conditional and unconditional variance, financial econometrics literature in the field of conditional variance models has witnessed an extraordinary growth, both in theory and in applications, with the addition of new and more powerful extensions, e.g., the GARCH model by Bollerslev (1986) and the EGARCH model by Nelson (1991), which have dramatically improved the fitting performances of the initial ARCH formulation.

Taylor (1986), on the other hand, offers an alternative with which to model the conditional variance of financial returns, the stochastic variance model. Together with its extensions (Harvey et al. [1994], Harvey and Shephard [1996], Sandmann and Koopman [1998], Jacquier et al. [2001], and Chib et al. [2001]), the proposed SV model captures more qualitative features of financial returns. In comparison with ARCH-GARCH models, which consider conditional variances as a deterministic function of past returns, the SV models describe variance (or rather the log-conditional variance) as a stochastic latent process, which however can be estimated. Besides, ARCH-GARCH can be easily estimated by the Maximum Likelihood and Quasi Maximum Likelihood techniques, while, in the SV framework, the presence of the latent variable makes estimation a harder task.

These difficulties however have boosted research into estimation techniques, and now it is possible to choose among different estimation methodologies: Generalized Method of Moments (Andersen and Sørensen [1996]), which is an evolution of the earlier Moments Matching applied by Taylor (1986; 1994) and Quasi Maximum Likelihood (Ruiz [1994], Harvey et al. [1994] and Harvey and Shephard [1996]). In addition, a number of simulation methodologies can be used: Importance Sampling (Danielsson and Richard [1993]), Indirect Inference (Gouriéroux et al. [1993]), Efficient Method of Moments (Gallant and Tauchen [1996]), Monte Carlo Likelihood (Durbin and Koopman [1997], Sandmann and Koopman [1998]), and Monte Carlo Markov Chain (Jacquier et al. [1994 and 2001], Kim et al. [1998] and Chib et al. [2001]). The first group of techniques for estimating SV models (GMM and QML) are easy to apply and less time-consuming, while the latter (IS, II, EMM, MCL, MCMC) sometimes appear to be 
more efficient but are often extremely time-consuming.

The need for conditional variance models to help to ensure the best fit to empirical data is due to the ever-increasing importance which variance plays in modern financial theory and applications, in particular in option pricing, risk management, the construction of optimal assets portfolios and other issues.

After analyzing and comparing at a theoretical level the two classes of models, ARCH-GARCH and SV, they are fitted to the data and the empirical results are compared, to contribute to the identification of the best data-fitting model among the various ARCH-GARCH and SV formulations considered here. In particular, for each class, three formulations are chosen: a standard version, a fat-tailed and an asymmetric one. For GARCH, this turns out to be the comparison of GARCH(1,1) (Bollerslev [1986]), GARCH(1,1)-t (Bollerslev [1987]) and EGARCH(1,1) (Nelson [1991]) models. For the SV models, the SV standard (Taylor [1986]), the SV-t (Harvey et al. [1994]) is compared to the asymmetric SV (Harvey and Shephard [1996]). For the empirical strategy to verify goodness of fit some diagnostic tests are carried out for each class of models and between the two classes.

This paper is organized as follows: Section II examines the theoretical underpinnings and estimation of the GARCH and SV type models. Section III discusses the application of the aforementioned models using financial time series data. Section IV presents the summary of the results and conclusions.

\section{Some Theoretical Aspects}

\section{A. Introduction to Conditional Variance Models}

The econometric models most frequently applied to describe the stylized facts of financial returns are based on the following general equation (Taylor [1986], Harvey and Shephard [1993], Bollerslev et al. [1994], Ghysels et al. [1996], Campbell et al. [1997])

$$
r_{t}=\mu_{t}+\xi_{t} \sigma_{t}
$$

where $r_{t}$ stands for the return at time $t, \xi_{t}$ is an IID random variable with zero mean and unitary variance, and $\sigma_{t}$ is volatility at time. The variable $\mu_{t}=\mu\left(I_{t}\right)$ is a function of $I_{t}$, the information set available at time $t$ 
(Andersen [1994]). It represents the trend (Ghysels et al. [1996]) or rather, the mean or the level of the returns data generating process (DGP). Usually, when returns are not correlated, $\mu_{t}$ is simply the sample mean of the returns. In order to represent a possible linear structure, the stochastic processes most frequently used are the ARMA (Box and Jenkins [1976]). The mean $\mu_{t}$ can also include exogenous variables (Bollerslev [1986], Harvey and Shephard [1993], Sandmann and Koopman [1998), such as seasonal and days dummies or trading volumes (Tauchen et al. [1996], Lamourex and Lastrapes [1994]). By subtracting $\mu_{t}$ from $r_{t}$ it follows that

$$
y_{t}=\xi_{t} \sigma_{t}
$$

where $y_{t}=r_{t}-\mu_{t}$. The main assumption in equation (2) is that $y_{t}$ is a white noise, and for this reason $y_{t}$ is defined as the pre-whitened returns series. With reference to equation (1), there are two possible sources of variability in the model: the mean $\mu_{t}$ and volatility $\sigma_{t}$. Usually, the mean is smaller in magnitude than volatility, suggesting that most of the variability is due to the latter.

Let us now consider a non-linear transformation (call it $f$ ) of volatility $\sigma_{t}$, which is represented as a function of past information $I_{t}$,

$$
f\left(\sigma_{t}\right)=g\left(I_{t}\right)
$$

Conditional on $I_{t}$, the main assumption is that $y_{t}$ is distributed as normal,

$$
y_{t} \mid I \sim N\left(0, \sigma^{2}\right)
$$

Given the general model described by equations (1) and (3) and assumption (4), two different types of models can recognized, related to the different way in which the functions $f$ and $g$ are specified and parameterized (Shephard [1996]):

Observation-driven models, also defined by Taylor (1986) as models in which the variance changes are caused by past prices, where $f\left(\sigma_{t}\right)$ is a function of the realized returns until $t$.

Parameter-driven models, otherwise defined as models in which the variance changes are not caused by prices (Taylor [1986]), where 
$f\left(\sigma_{t}\right)$ depends on a latent or unobserved variable.

In the first class are the generalized autoregressive heteroskedasticity models (GARCH and variants), while more frequently used among the second class are the Stochastic Volatility (SV) models.

For the GARCH models the function $f$ is usually specified as the square of volatility $\sigma_{t}, g$ is structured as an ARMA process and includes past squared returns and possibly past conditional variances (or log-variances for the EGARCH). In this case, $y_{t} \mid\left(I_{t}=Y_{t}\right)$ is distributed as $N\left(0, \sigma^{2}\right)$ (Engle [1982], Bollerslev [1986], Nelson [1991]), where $I_{t}$ is composed of the set of returns observed until time $t-1$.

The SV models $f=\ln \left(\sigma_{t}^{2}\right)$ is the logarithm of the conditional variance,(as in the EGARCH specification) and $g$ is usually specified as the sum of an AR(1) process and a random error. Thus, the SV model considers volatility as a stochastic variable. The conditional distribution of $y_{t}$ is then the following (Taylor [1994]):

$$
y_{t} \mid\left(I_{t}=h_{t}\right) \sim N\left(0, \exp \left(h_{t}\right)\right)
$$

where $I_{t}$ is log-volatility, $h_{t}=\sigma_{t}^{2}$, regarded as a latent variable which can be estimated on the basis of the observations on returns.

\section{B. GARCH Models}

Referring to equation (2), in the $\operatorname{GARCH}(p, q)$ model the conditional variance is characterized by

$$
\sigma_{t}^{2}=\alpha_{0}+\sum_{i=1}^{q} \alpha_{i} y_{t-i}^{2}+\sum_{j=1}^{p} \beta_{j} \sigma_{t-j}^{2}
$$

where $p$ refers to the lags of the variable $\sigma_{t}^{2}$ and $q$ to the lags of the variable $y_{t}$. When $p=1$ and $q=1$, the $\operatorname{GARCH}(1,1)$ model is obtained,

$$
\sigma_{t}^{2}=\alpha_{0}+\alpha_{1} y_{t-1}^{2}+\beta_{1} \sigma_{t-1}^{2}
$$

The GARCH model may be generalized by letting $\xi_{t}$ in equation (2) have a student $t$ distribution with $v$ degrees of freedom. The importance of this class of models, known as GARCH- $t$ (Bollerslev [1987]), is due to the fact that they are able to capture the excess kurtosis present in many financial time series. 
The $\operatorname{EGARCH}(p, q)$ model, on the other hand, considers the logarithm of the conditional variance as follows

$$
\ln \left(\sigma_{t}^{2}\right)=\alpha_{0}+\sum_{i=1}^{q} \alpha_{i}\left(\left|\xi_{t-i}\right|-E\left(\left|\xi_{t-i}\right|+\gamma \xi_{t-i}\right)\right)+\sum_{j=1}^{p} \beta_{j} \ln \left(\sigma_{t-j}^{2}\right)
$$

where $\xi_{t}$ are the standardized residuals. This model allows us to describe the asymmetric effect which is often present in the returns series. As explained by Nelson (1991), if $\alpha_{i}>0$ a deviation of $\xi_{t}$ from its expected value causes the conditional variance of $y_{t}, \sigma_{t}^{2}$, to be bigger than it otherwise would be. The parameter $\gamma$ allows this effect to be asymmetric: if $\gamma=0$, a positive $\xi_{t}$ will have the same effect on volatilities produced by a negative $\xi_{t}$ of the same magnitude. But if $-1<\gamma<0$, a positive $\xi_{t}$ will increase future volatilities less than a negative $\xi_{t}$. Finally, if $\gamma<-1$, a positive $\xi_{t}$ will reduce future volatilities while a negative one will produce the opposite effect.

\section{Stochastic Volatility Models}

The basic stochastic volatility model is

$$
\begin{gathered}
y_{t}=e^{h_{t} / 2} \xi_{t} \\
h_{t}=\alpha+\phi h_{t-1}+\eta_{t}
\end{gathered}
$$

where $\eta_{t} \sim N I D\left(0, \sigma_{\eta}^{2}\right), h_{t}=\ln \left(\sigma_{t}^{2}\right)$ is the volatility function and working with logarithms ensures that $\sigma_{t}^{2}$ is always positive; $\xi_{t}$ is a white noise process with unit variance generated independently of $\eta_{t}$ and $|\phi|<1$, which ensures the strict stationarity of the process. It follows that $h_{t}$ is stationary with mean $\alpha_{h}=\alpha /(1-\phi)$ and variance $\sigma_{h}{ }^{2}=\sigma_{\eta}{ }^{2} /\left(1-\phi^{2}\right)$.

Despite a very parsimonious representation, this model captures most of the empirical regularities found in financial time series (Ghysels et al. [1996]). The component $\sigma_{t}$ is known in financial literature as volatility or conditional variance (see, for istance, Engle [1982], Bollerslev [1986], Taylor [1994]). In particular, one interpretation of the process $h_{t}$, which has its origin in Clark (1973) and is refined in Tauchen and Pitts (1983), is that stochastic volatility reflects the random and uneven flow of new information to the financial markets. 
Transforming $y_{t}$ of equation (8) by taking logarithms of the squares, the following measurement equation of a linear state space model is obtained

$$
\ln y_{t}^{2}=E\left(\ln \xi_{t}^{2}\right)+h_{t}+\varepsilon_{t}
$$

where $\varepsilon_{t}=\ln \left(\xi_{t}^{2}\right)-E\left(\ln \left(\xi_{t}^{2}\right)\right)$. If $\xi_{t}$ is distributed as a standard normal, $\ln \left(\xi_{t}\right)$ follows a non-Gaussian distribution with mean $\psi(1 / 2)-\ln (1 / 2) \approx$ -1.27 and variance $\pi^{2} / 2$ (Ruiz [1994], Sandmann and Koopman [1998]) where $\psi(\cdot)$ is the digamma function (Abramovitz and Stegun [1970]).

Equation (8) may be written as

$$
y_{t}=\beta e^{h_{t} / 2} \xi_{t}
$$

where $\beta$ is a scale parameter that allows the state space model to be rewritten as

$$
\begin{gathered}
\ln y_{t}^{2}=\omega+h_{t}+\varepsilon_{t} \\
h_{t}=\phi h_{t-1}+\eta_{t}
\end{gathered}
$$

where $\omega=\ln \left(\beta^{2}\right)+E\left(\ln \left(\xi_{t}\right)\right)$. As $\ln \left(\beta^{2}\right)=\alpha /(1-\phi)$, if the $\phi$ and $\beta$ parameters are known, it is easy to obtain the value of $\alpha$.

When $\xi_{t}$ has a student $t$ distribution with $v$ degrees of freedom, equation (8) represent a SV-t model. It can be shown (see, Harvey et al. 1994) that when $h_{t}$ is stationary, $y_{t}$ is white noise and from the properties of the $t$ distribution it follows that the unconditional variance generalizes to

$$
\left(\frac{v}{v-2}\right) e^{\alpha_{h}+\sigma_{h}^{2} / 2}
$$

In this case $\xi_{t}$ of the equation (8) may be written

$$
\xi_{t}=\frac{s_{t}}{\sqrt{\kappa_{t}}}
$$

for $t=1, \ldots, T$, where $\zeta_{t}$ is a standard normal variate and $v \kappa_{t}$ is distributed, independently of $\zeta_{t}$ as a $\chi^{2}$ with $v$ degrees of freedom. Thus 


$$
\ln \left(\xi_{t}^{2}\right)=\ln \left(\zeta_{t}^{2}\right)-\ln \left(\kappa_{t}\right)
$$

and it follows that the mean and variance of $\ln \left(\kappa_{t}\right)$ are respectively $\psi^{\prime}(v / 2)-\ln (v / 2)$ and $\psi^{\prime}(v / 2)$, where $\psi^{\prime}(\cdot)$ is the trigamma function (Abramovitz and Stegun [1970]).

When there is a dependence between $\xi_{t}$ and $\eta_{t}$ the model picks up the kind of asymmetric behavior that is often found in stock prices (Schwert [1989], Nelson [1991], Engle and Ng [1993], Nicolis [2000]). The asymmetry refers to the impact of negative returns on predicted volatility with respect to the impact of positive ones. For example, a negative return tends to be associated with an increase in predicted volatility, suggesting a negative correlation between $\xi_{t}$ and $\eta_{t}$ of equations (8) and (9).

The linear state space form (13) can be modified to estimate asymmetric models. Harvey and Shephard (1996) observed that, even if $\xi_{t}$ and $\eta_{t}$ in the equation (8), respectively, and equation (9) are correlated, the disturbances in the linear state space form are uncorrelated provided the joint distribution of $\xi_{t}$ and $\eta_{t}$ is symmetric. Hence, as in the state-space transformation, taking the square of the observations, the information on the dependence between $\xi_{t}$ and $\eta_{t}$ is lost. Harvey and Shephard (1996) showed that this information can be recovered by conditioning $s_{t}$, which is the sign of the observations, which of course is the same as $\xi_{t}$. The linear state space form including the asymmetric component becomes the following

$$
\begin{gathered}
\ln y_{t}^{2}=\omega+h_{t}+\varepsilon_{t} \\
h_{t+1}=\phi h_{t}=s_{t} \mu^{*}+\eta_{t}^{*} \\
{\left[\begin{array}{c}
\varepsilon_{t} \\
\eta_{t}^{*}
\end{array}\right] \mid s_{t} \sim I I D\left(\left[\begin{array}{l}
0 \\
0
\end{array}\right],\left[\begin{array}{cc}
\sigma_{\varepsilon}^{2} & \gamma^{*} s_{t} \\
\gamma^{*} s_{t} & \sigma_{\eta}^{2}-\mu^{* 2}
\end{array}\right]\right)}
\end{gathered}
$$

where $\mu^{*}$ denotes the expectation taken conditional on $\xi_{t}$ being positive, $\mu^{*}=E_{+}\left(\eta_{t}\right)$, and $\gamma^{*}$ assigns a similar interpretation to variance and covariance operators, $\gamma^{*}=\operatorname{cov}\left(\eta_{t}, \varepsilon_{t}\right)$.

\section{Estimation}


It is very quick and easy to estimate GARCH models through maximum likelihood or quasi maximum likelihood via iterative numerical algorithms (see, Engle [1982], Bollerslev [1986]). On the contrary, the estimation procedure of SV is a very difficult task because the vector of latent observations $\left\{h_{t}\right\}$, for $t=1,2, \ldots, T$, has to be integrated out of the joint density of the volatility and returns in one $T$-dimensional integration,

$$
\ell(\Omega)=\int_{R^{T}} f(y, h \mid \Omega) d h
$$

to obtain the likelihood function $\ell(\Omega)$, where $\Omega$ is the vector of the parameters $\left(\alpha, \sigma_{\eta}, \phi\right)$. This integral does not have an analytical solution.

So numerical methods (which are often very computer-intensive and quite difficult to implement) must be employed for evaluation. This is mainly what renders the $\mathrm{SV}$ formulation less attractive than the GARCH one, and explains why the latter has been more widely adopted. On the other hand, the computational problems it generates have created a challenge for researchers to develop more efficient and computer-intensive estimation methods such as QML, GMM, EMM and MCMC (for a survey on a comparison of estimation techniques, see Ghysels et al. [1996], Shephard [1996], Jacquier et al., [1994], Sandmann and Koopman [1998] and Andersen et al. [1999]). In this work, the QML approach based on the Kalman filter is considered for estimating the different types of SV models (Harvey et al. [1994], Ruiz [1994], Harvey and Shephard [1996]). The chosen estimation method represents a good compromise between efficiency and computational time consumption.

Considering the equation (8) when $\xi_{t} \sim N I D(0,1)$ if $y_{t}$ is squared and logged, the model can be rewritten as

$$
\begin{gathered}
\ln y_{t}^{2}=-1.27+h_{t}+\varepsilon_{t} \\
h_{t}=\alpha+\phi h_{t-1}+\eta_{t}
\end{gathered}
$$

where $\varepsilon_{t}=\ln \left(\xi_{t}^{2}\right)-E\left(\ln \left(\xi_{t}^{2}\right)\right)$ follows the $\chi_{1}^{2}$ distribution with mean zero and variance $\pi^{2} / 2$. The QML method approximates the distribution of $\mathcal{E}_{t}$ by $N I D\left(0, \pi^{2} / 2\right)$. The system (20) represents the measurement and 
transition equations of the general linear state model in which the state space form relates an observed time series $y_{t}$ to an unobserved state vector $h_{t}$. Using Kalman filter the Gaussian likelihood by the prediction error decomposition is obtained. The parameter estimates of $\omega=(\alpha, \phi$, $\left.\sigma_{\eta}^{2}\right)$ result from numerical optimization.

The standard theory for the estimation of unobserved component time series models with non-normal errors applies to the estimate of $\omega$ (Ruiz [1994]).

When $\xi_{t}$ in equation (8) is a student $t$ variable with $v$ degrees of freedom as given by (14), the variance of of the model (20) is $\sigma_{\varepsilon}^{2}=\pi^{2} / 2$ $+\psi^{\prime}(v / 2)$. The estimated parameter set becomes $\omega^{*}=\left(\alpha, \phi, \sigma_{\eta}^{2}, \sigma_{\varepsilon}^{2}\right)$ with the restriction $\sigma_{\varepsilon}^{2} \geq \pi^{2} / 2$. The estimates of $\omega^{*}$ can be obtained by the QML procedure approximating the distribution of $\varepsilon_{t}$ by $N I D\left(0, \sigma_{\varepsilon}^{2}\right)$, and maximizing the resulting quasi-likelihood function (see Ruiz [1994], for the asymptotic theory).

Let us consider now the estimation of the asymmetric SV model. Assuming that the joint distribution of $\xi_{t}$ and $\eta_{t}$ is symmetric, the disturbances in the linear state space form of (16) and (17) are uncorrelated. For this, Harvey and Shephard (1996) suggest the "unrestricted" QML method for estimating the parameters $\omega, \phi, \sigma_{\eta}^{2}, \sigma_{\varepsilon}^{2}$, $\mu^{*}$ and $\gamma^{*}$. The QML estimators are obtained by treating $\eta_{t}$ and $\varepsilon_{t}$ as though they were normal and maximizing the prediction-error decomposition form of the likelihood achieved via the Kalman filter. This procedure estimates the parameters without any distributional assumption, apart from the existence of fourth moments of $\eta_{t}$ and $\xi_{t}$. As a consequence it doesn't provide an estimate of the parameters of the joint distribution of $\eta_{t}$ and $\xi_{t}$ which is denoted by $\rho$. Such an estimate can be constructed by making a distributional assumption about $\xi_{t}$ and as well as $\eta_{t}$.

When the joint distribution of $\eta_{t}$ and $\xi_{t}$ is bivariate normal with correlation $\rho, E\left(\eta_{t} \mid \xi_{t}\right)=\rho \sigma_{\eta} \xi_{t}$, thus

$$
\mu^{*}=\rho \sigma_{\eta} \sqrt{2 / \pi}=0.7979 \rho \sigma_{\eta} .
$$

Moreover,

$$
\gamma^{*}=\rho \sigma_{\eta} E\left(\left|\xi_{t}\right| \ln \xi_{t}\right)-.7979 \rho \sigma_{\eta} E\left(\ln \xi_{t}^{2}\right)=1.1061 \rho \sigma_{\eta}
$$


(see Harvey and Shephard [1996], for detailed results). As for the standard model, it can be shown that the QML estimator is consistent and asymptotically normal. Even if conditioning on the sign $s_{t}$ of the observations complicates matters somewhat in that the covariance between the two disturbances in equation (18) varies according to $s_{t}$, consistency and asymptotic normality can nonetheless be demonstrated (Harvey and Shephard [1996]).

\section{E. Differences and Analogies}

From sub-sections $\mathrm{A}$ and $\mathrm{C}$ it stands out that the fundamental difference between the GARCH and the SV models consists of a different specification of the equation describing the conditional variance. Other important differences refer to the sources of variability, restrictions on parameters, stationarity conditions, unconditional kurtosis and estimation techniques.

\section{Sources of variability and restrictions on parameters}

The GARCH models have only one source of variability which stands for the noise in equation (1), while $S V$ is characterized by two separate sources of variability: the noise $\xi_{t}$ in equation (8) and innovation $\eta_{t}$ in the volatility equation (9). The latter makes the conditional variance a stochastic process and determines the degree of mixing of the returns. In the GARCH class case, in particular when adopting a high order of parameters, increasing problems in managing and interpreting parameter estimates have to be tackled. Besides, it takes a lot of restrictions on parameter values to guarantee that, for each $t$ the conditional variances $\sigma_{t}^{2}$ are non-negative. In the EGARCH formulation the problem is solved considering the logarithm of the variance. When fitting the SV model, only a few restrictions are necessary to perform estimation, and the parameter meanings are easy to interpret. As in the EGARCH formulation, the SV model describes the logarithm of the variance, and no other parametric bounds are required in order to estimate the model.

\section{Parameterization and Stationarity Conditions}

The squared returns of a generic GARCH model can be re-parameterized as an ARMA process (Bollerslev [1986]). The squared returns of the SV model behave like the ARMA (more precisely, an $\operatorname{ARMA}(1,1))$ as well, especially as $\exp \left(\sigma_{t}^{2}\right) /\left(1-\phi^{2}\right) \rightarrow 0$ and $\phi \rightarrow 1$ (Taylor [1986]). For this reason, the $\phi$ parameter takes on the same 
interpretation of $\Sigma \alpha_{i}+\Sigma \beta_{j}$, that is a measure of volatility persistence. Moreover, in order to ensure the (weak) stationarity of the conditional variance (and hence of the returns), the GARCH models require that $\Sigma \alpha_{i}+\Sigma \beta_{j}<1$ while the SV class needs $|\phi|<1$.

\section{Unconditional kurtosis}

The widely used GARCH specification in the conditional variance models literature, i.e., the $\operatorname{GARCH}(1,1)$ with normal conditional distribution, is adequate to describe returns of conditional heteroskedasticity, but it shows major limits when representing the high kurtosis of unconditional distributions of return (Geweke [1994], Shephard [1996], Starica and Pictet [1997], Kim et al. [1998]). Bai et al. (2001) show that $y_{t}$ 's unconditional kurtosis can be broken down into two components: kurtosis induced by the persistence parameters $\alpha_{1}+\beta_{1}$, and kurtosis generated by the distribution of noise $\xi_{t}$, the two components acting in a symmetric and interactive way to determine overall kurtosis. In particular, the authors prove that, for the $\operatorname{GARCH}(1,1)$ with $\xi_{t} \sim N(0,1)$, no contribution to the unconditional kurtosis arises from the innovation distribution, but that for the values of $\alpha_{1}+\beta_{1}$ more frequently found in empirical applications, that is 0.85 $<\alpha_{1}+\beta_{1}<1$, the induced kurtosis is too small to replicate the high unconditional kurtosis. Thus Bollerslev (1987), Nelson (1991), Shephard (1996), Terasvirta (1996), Mikosch and Starica (2000), among others, suggest the use of GARCH processes with fat-tailed errors, like $t$ student or GED. In the SV case, the kurtosis coefficient of the unconditional returns has the following expression (Taylor [1986], Taylor [1994], Liesenfeld and Jung [2000], Bai et al. [2001])

$$
\kappa=E\left(\varepsilon_{t}^{4}\right) e^{\sigma_{h}^{2}}
$$

Equation (23) shows that the unconditional distribution kurtosis of $y_{t}$ is the result of two separate components which, as in the GARCH case, operate symmetrically and interactively: conditional kurtosis, depending on the innovation $\xi_{t}$ distribution, and kurtosis induced by the volatility unconditional variance, $\sigma_{h}{ }^{2}=\sigma_{\eta}{ }^{2} /\left(1-\phi^{2}\right)$, which in turn depends on the persistence parameter $\phi$. Nevertheless, unlike the GARCH models, if $\xi_{t}$ $\sim N(0,1)$, conditional kurtosis is equal to 3 , and thus, since 
$e^{\sigma_{h}^{2}}>0, \kappa>0$, in (23). As a consequence, the SV model can be defined with normal conditional distribution as a thick-tailed model. However, Liesenfeld and Jung (2000) show that for the persistence values most likely in empirical applications, i.e., with $\phi>0.9$, the normal SV model does not fit well enough to capture the entire unconditional kurtosis, this being consistent with results obtained by Geweke (1994), Terasvirta (1996), Gallant et al. (1997), among others. So, for the SV as well, it is necessary to adopt heavy-tailed conditional distributions. Moreover, in the GARCH models, the fourth moment may not exist, in which case it is not possible to calculate unconditional kurtosis. On the contrary, in the SV models, the fourth moment exists whenever $h_{t}$ is a stationary process: thus it is always possible to calculate kurtosis.

\section{Conditional Variance Asymmetry}

For the EGARCH formulation He et al. (1999) show that, under specific assumptions of the expected values of certain functions of returns innovation, even when $\varepsilon_{t} \sim N(0,1)$, unconditional kurtosis is $\kappa>3$ and depends on parameter values. Although EGARCH returns are more fat-tailed than GARCH ones, the normal distribution can not describe the entire unconditional kurtosis. As for the standard SV formulation, equation (23) is still valid in the asymmetric case.

\section{Estimation Algorithms}

The method usually utilized for estimating the conditional variance models is the QML technique. More precisely, for the GARCH model class it easy to maximize the likelihood function via iterative numerical algorithms. Instead, in the SV model class it is not possible to analytically solve the likelihood function which is T-dimensional integrated. For this, as already seen in section II.C, it is necessary to use a more complex procedure based on the Kalman Filter (Harvey and Shephard [1996]).

\section{Empirical Application}

\section{A. Dataset}

To implement empirical comparisons a series of four indices are considered: (1) Deutsche Aktienindex (DAX): 2119 observations, from 
TABLE 1. Main Descriptive Statistics

\begin{tabular}{lcclcrr}
\hline & Mean $\times 10^{3}$ & St. dev. $\times 10^{3}$ & Skewness & Kurtosis & JB & LB(30) \\
\hline DAX & 0.654 & 12.962 & -0.430 & 5.677 & 703 & 1071 \\
Dow Jones & 0.565 & 9.832 & -0.460 & 8.058 & 2359 & 552 \\
FTSE100 & 0.333 & 9.713 & -0.160 & 4.389 & 187 & 1537 \\
MIB30 & 0.627 & 14.986 & -0.073 & 4.415 & 179 & 935 \\
\hline
\end{tabular}

TABLE 2. LM Test Statistics and Their Critical Values

\begin{tabular}{rlcccr}
\hline Lag & DAX & Dow Jones & FTSE100 & MIB30 & \multicolumn{1}{c}{$\chi^{2}$} \\
\hline 1 & 139.37 & 91.374 & 58.815 & 100.705 & 3.841 \\
5 & 221.207 & 164.874 & 219.74 & 225.011 & 11.071 \\
30 & 293.7 & 228.431 & 333.178 & 304.026 & 43.773 \\
50 & 322.599 & 251.802 & 359.503 & 325.319 & 67.505 \\
\hline
\end{tabular}

Note: For the details on LM tests, see Engle (1982). The $\chi^{2}$ critical values at at the $5 \%$

04/01/1993 to 01/06/2001; (2) Dow Jones (DJ): 2123 observations, from 04/01/1993 to 01/06/2001; (3) Financial Times Stock Exchange (FTSE100): 2124 observations, from 04/01/1993 to 01/06/2001; (4) Milano Indice Borsa (MIB30): 2124 observations, from 04/01/1993 to 01/06/2001.

The choice of these indices is due to the fact that they represent the different stock exchange markets. The returns from the index series collected each trading day at closing time are used. The values are the continuously compounded returns calculated as the natural logarithm of two consecutive index values, $r_{t}=\ln \left(P_{t} / P_{t-1}\right)$, a transformation used to obtain approximately stationary series. Returns are then filtered through suitable ARMA processes to eliminate or reduce any data linear structure. Residuals (or adjusted returns) obtained are approximately distributed as white noise.

Table 1 presents several descriptive statistics of the data. The kurtosis values indicate that the sample distributions of the adjusted returns have heavy tails, especially for Down Jones. In addition, the Jarque-Bera (1987) tests clearly show non-normality of distribution of the four indices. The Ljung-Box (1978) tests (LB test) show strong autocorrelations in the squared returns distributions. The Engle (1982) 
TABLE 3. Asymmetry Tests

\begin{tabular}{lcccccc}
\hline & $\begin{array}{l}\text { Global } \\
\text { standard } \\
\text { deviation } \\
\sigma\end{array}$ & $\begin{array}{l}\text { Positive } \\
\text { standard } \\
\text { deviation } \\
\sigma^{+}\end{array}$ & $\begin{array}{l}\text { Negative } \\
\text { standard } \\
\text { deviation } \\
\sigma^{-}\end{array}$ & $\begin{array}{l}\text { Absolute } \\
\text { percentage } \\
\text { difference } \\
\Delta \%\end{array}$ & $\begin{array}{l}\text { Number } \\
\text { of positive } \\
m\end{array}$ & $\begin{array}{c}\text { Number } \\
\text { of negative } \\
n\end{array}$ \\
\hline DAX & 0.013 & 0.0118 & 0.0142 & 20.34 & 1160 & 957 \\
Dow Jones & 0.0098 & 0.0091 & 0.0106 & 16.48 & 1141 & 978 \\
FTSE100 & 0.0097 & 0.0093 & 0.0102 & 9.68 & 1109 & 1011 \\
MIB30 & 0.015 & 0.0148 & 0.0153 & 3.38 & 1080 & 1035 \\
\hline
\end{tabular}

Note: For the details on the tests see Drobetz and Zimmermann (2003).

LM test in table 2 indicates the presence of $\mathrm{ARCH}$ effects in the conditional variance at 1, 5, 30 and 50 lags.

Table 3 shows the results of the procedure suggested by Drobetz e Zimmermann (2003) for detecting the presence of asymmetry in financial returns series and performed before fitting any model to the data. In short, the technique requires the splitting of each dataset into two separate subsets, the positive returns subset $\left(r_{t}^{+}\right)$and the negative returns subset $\left(r_{t}^{-}\right)$. Applying the following equations, the standard deviation for each subset minus the whole dataset mean value is calculated:

$$
\begin{gathered}
\sigma^{+}=\sqrt{\frac{1}{m-1} \sum_{t=1}^{m}\left(r_{t}^{+}-\frac{1}{T} \sum_{t=1}^{T} r_{t}\right)^{2}} \\
\sigma^{-}=\sqrt{\frac{1}{n-1} \sum_{t=1}^{n}\left(r_{t}^{-}-\frac{1}{T} \sum_{t=1}^{T} r_{t}\right)^{2}}
\end{gathered}
$$

where $m$ and $n$ represent the number of observations for each subset. The quantity $\Delta \%$ indicates the absolute percentage difference between $\sigma^{+}$and $\sigma^{-}$.

If the positive subset standard deviation is smaller (bigger) than the negative (positive) subset, asymmetry is likely to be present in the adjusted returns datasets, i.e. bad news and good news with the same absolute value have a different impact on future volatility (Bali [2000], Engle and $\mathrm{Ng}$ [1993]). It is possible to briefly conclude that the four 
TABLE 4. GARCH(1,1) Estimates

\begin{tabular}{ccccc}
\hline Estimates & DAX & Dow Jones & FTSE100 & MIB30 \\
\hline \multirow{2}{*}{$\alpha_{0}$} & $2.72 \times 10^{-4}$ & $8.16 \times 10^{-7}$ & $4.51 \times 10^{-7}$ & $1.32 \times 10^{-5}$ \\
& $\left(4.96 \times 10^{-5}\right)$ & $\left(2.05 \times 10^{-7}\right)$ & $\left(1.71 \times 10^{-7}\right)$ & $\left(3.01 \times 10^{-6}\right)$ \\
& {$[5.48]$} & {$[4.20]$} & {$[2.64]$} & {$[4.38]$} \\
$\alpha_{1}$ & 0.089 & 0.083 & 0.045 & 0.117 \\
& $(0.010)$ & $(0.007)$ & $(0.007)$ & $(0.017)$ \\
$\beta_{1}$ & {$[8.55]$} & {$[12.07]$} & {$[6.61]$} & {$[7.08]$} \\
& 0.895 & 0.912 & 0.951 & 0.824 \\
& $(0.011)$ & $(0.007)$ & $(0.007)$ & $(0.025)$ \\
& {$[77.9]$} & {$[118.3]$} & {$[134.7]$} & {$[32.7]$} \\
\hline
\end{tabular}

Note: Parentheses include the standard errors and brackets the t-values of the estimates.

TABLE 5. GARCH(1,1)- $t$ Estimates

\begin{tabular}{ccccc}
\hline Estimates & DAX & Dow Jones & FTSE100 & MIB30 \\
\hline \multirow{2}{*}{$\alpha_{0}$} & $1.29 \times 10^{-4}$ & $5.10 \times 10^{-7}$ & $3.61 \times 10^{-7}$ & $1.04 \times 10^{-5}$ \\
& $\left(5.34 \times 10^{-5}\right)$ & $\left(2.04 \times 10^{-7}\right)$ & $\left(1.90 \times 10^{-7}\right)$ & $\left(2.94 \times 10^{-6}\right)$ \\
\multirow{4}{*}{$\alpha_{1}$} & {$[2.41]$} & {$[2.50]$} & {$[1.90]$} & {$[3.54]$} \\
& 0.062 & 0.046 & 0.042 & 0.098 \\
$\beta_{1}$ & $(0.010)$ & $(0.008)$ & $(0.008)$ & $(0.028)$ \\
& {$[5.99]$} & {$[5.85]$} & {$[5.60]$} & {$[5.72]$} \\
& 0.915 & 0.929 & 0.948 & 0.833 \\
& $(0.013)$ & $(0.011)$ & $(0.009)$ & $(0.028)$ \\
& {$[68.3]$} & {$[84.96]$} & {$[103.2]$} & {$[29.3]$}
\end{tabular}

Note: Parentheses include the standard errors and brackets the t-values of the estimates.

data sets analyzed show the stylized facts typical of financial returns series: heavy tails, persistence, heteroskedasticity, strong sample autocorrelations in the squared returns distribution, and asymmetry.

\section{B. Estimation of GARCH Models}

Now the results of the empirical applications of the models described in section I are shown to represent the qualitative features of the financial returns datasets. In particular, three different formulations of the GARCH family are fitted: the standard $\operatorname{GARCH}(1,1)$ with normal conditional distribution, the GARCH(1,1)- $t$ with $t$ student conditional distribution, also known as the fat-tailed GARCH, and the 
TABLE 6. EGARCH(1,1) Estimates

\begin{tabular}{ccccc}
\hline Estimates & DAX & Dow Jones & FTSE100 & \multirow{2}{*}{ MIB30 } \\
\hline \multirow{2}{*}{$\alpha_{0}$} & -0.309 & -0.524 & -0.247 & -1.053 \\
& $(0.035)$ & $(0.054)$ & $(0.040)$ & $(0.172)$ \\
$\alpha_{1}$ & {$[-8.94]$} & {$[-9.66]$} & {$[-6.13]$} & {$[-6.11]$} \\
& 0.206 & 0.195 & 0.115 & 0.260 \\
& $(0.022)$ & $(0.020)$ & $(0.018)$ & $(0.030)$ \\
$\gamma$ & {$[9.44]$} & {$[9.62]$} & {$[6.22]$} & {$[8.63]$} \\
& -0.271 & -0.499 & -0.529 & -0.172 \\
$\beta_{1}$ & $(0.052)$ & $(0.088)$ & $(0.112)$ & $(0.053)$ \\
& {$[-5.19]$} & {$[-5.66]$} & {$[4.71]$} & {$[-3.28]$} \\
& 0.965 & 0.960 & 0.983 & 0.900 \\
& $(0.005)$ & $(0.005)$ & $(0.003)$ & $(0.019)$ \\
\hline
\end{tabular}

Note: Parentheses include the standard errors and brackets the t-values of the estimates.

$\operatorname{EGARCH}(1,1)$, with normal conditional distribution. The autoregressive and moving averages of GARCH components have been chosen by AIC, Akaike Information Criterion (Akaike [1974]).

Tables 4, 5 and 6 report the estimates and their standard deviations of GARCH( $(1,1)$, GARCH(1,1)- $t$ and EGARCH( 1,1$)$ models. In general the parameter estimates are significantly different from zero except for the parameter $\alpha_{0}$ of the FTSE100 return series in the GARCH-t estimation.

Considering $\operatorname{GARCH}(1,1)$ and $\operatorname{GARCH}(1,1)-\mathrm{t}$, the sum $\alpha_{1}+\beta_{1}$ which represents volatility persistence, takes on high values (from 0.931 to 0.996 ).

Regarding EGARCH $(1,1)$ estimation, the parameter of asymmetry, $\gamma$, is negative in all series. This means that the impact of negative returns on expected volatility is greater than that of positive ones. In particular this impact is more significant for the Dow Jones and FTSE100 series. In order to assess and compare the goodness of fit for each GARCH type formulation fitted to the data, standardized returns are determined by volatility estimates $y_{t} / \sigma_{t}$ (it is the so called "devolatilization" procedure), and then standardized returns are tested to establish whether they are gaussian white noise or IID (see, Lundbergh and Terasvirta [2001] for a recent and thorough survey about GARCH diagnostic tests).

The Log-Likelihood values are displayed in table 7. Compared to 
TABLE 7. Log-Likelihood Ratio Statistics

\begin{tabular}{llll}
\hline & GARCH(1,1) & GARCH(1,1)- $t$ & EGARCH(1,1) \\
\hline DAX & $-32,619.9$ & $-32,584.4$ & $-32,609.6$ \\
Dow Jones & $-27,157.7$ & $-27,104.6$ & $-27,143.3$ \\
FTSE100 & $-27,170.3$ & $-27,161.3$ & $-27,170.6$ \\
MIB30 & $-28,227.0$ & $-28,212.1$ & $-28,231.5$ \\
\hline
\end{tabular}

TABLE 8. Kurtosis Values for Adjusted Returns and Standardized Residuals

\begin{tabular}{lcccc}
\hline & $y_{\mathrm{t}}$ & GARCH(1,1) & GARCH(1,1)- $t$ & EGARCH(1,1) \\
\hline DAX & 5.667 & 4.118 & 3.065 & 4.064 \\
Dow Jones & 8.058 & 4.707 & 3.018 & 4.466 \\
FTSE100 & 4.389 & 3.514 & 3.011 & 3.651 \\
MIB30 & 4.415 & 3.578 & 3.003 & 3.653 \\
\hline
\end{tabular}

the standard GARCH(1,1) model, Log-likelihood values point out that fat-tailed GARCH(1,1)- $t$ better estimate the returns of all four indexes. The EGARCH $(1,1)$ model appears better than the standard version only for the DAX and Dow Jones return series, while for FTSE100 it shows exactly the same fitting, the worst performance being for the MIB30 returns. In particular, it is evident that as long as the asymmetric effect (see table 7) weakens, the EGARCH performs as well as the standard one, and sometimes worse. Finally, it should be noted that between GARCH(1,1)- $t$ and $\operatorname{EGARCH}(1,1)$ the former always performs better. However, it is useful to remember that the formulation for the EGARCH model is different from that of GARCH and GARCH-t: the EGARCH models absolute shocks while the others model squared shocks. As a consequence comparison among the different likelihood function values is not very reliable. For this reason, in this paper work, alternative diagnostic tools such as the kurtosis values, the LB test for whiteness of residuals and the asymmetry test are utilized.

Table 8 shows kurtosis values for standardized filtered returns. $\operatorname{GARCH}(1,1)-t$ standardized returns have been "normalized" through the procedure suggested by Shephard (1996) and Kim et al. (1998) to compare models. Kurtosis estimates allow us to evaluate model adequacy to capture thick-tail unconditional distributions. For a perfect 
TABLE 9. LB Statistics for Standardized Residuals and Their Squared Values

\begin{tabular}{llll}
\hline & GARCH(1,1) & GARCH(1,1)- $t$ & EGARCH(1,1) \\
\hline$L B(30)$ & & & \\
DAX & 43.589 & 43.392 & 44.338 \\
Dow Jones & 38.437 & 37.652 & 44.317 \\
FTSE100 & 27.078 & 27.518 & 26.981 \\
MIB30 & 35.522 & 35.440 & 35.132 \\
$L B^{2}(30)$ & & & \\
DAX & 25.584 & 28.835 & 41.420 \\
Dow Jones & 15.038 & 20.814 & 22.033 \\
FTSE100 & 20.637 & 20.403 & 22.563 \\
MIB30 & 27.635 & 27.923 & 36.180 \\
\hline
\end{tabular}

Note: $L B$ is for statistics on standardized residuals and $L B^{2}$ is for their squared values. The critical value for the above LB statistics is $\chi^{2}(28)=41.337$.

fit, kurtosis should be equal to three (the gaussian distribution kurtosis). As before, GARCH(1,1)-t guarantees the best fit, capturing the whole unconditional kurtosis. EGARCH, as already explained in section II.B, performs better than the standard model, whenever asymmetry is strong, i.e., for DAX and Dow Jones, while no remarkable improvement when asymmetry is low.

The Ljung Box test for autocorrelations in simple standardized returns (see table 9), shows the presence of a residual linear structure not captured by any model for DAX returns. Concerning the other datasets, standardized residuals are distributed as white noise, except for the EGARCH model applied to Dow Jones returns. As for the squared standardized residuals, the LB test does not indicate any significant autocorrelations, but for the EGARCH model applied to DAX returns. In short, the GARCH standard and the GARCH- $t$ models guarantee a suitable goodness of fit, producing approximately IID standardized residuals, while the EGARCH model reveals some limits in fitting the data.

The Engle and $\mathrm{Ng}$ (1993) diagnostic tests are applied in order to check whether the EGARCH specification is adequate to describe returns asymmetry: the sign bias test (SBT) is a $t$-test which allows to verify the null hypothesis that $y_{t}^{2} / \hat{\sigma}_{t}^{2}$ are independent from $y_{t} / \hat{\sigma}_{t}$ signs; the negative size bias test (NSBT) and the positive size bias test (PSBT) are the $t$-tests of the null hypothesis that $y_{t}^{2} / \hat{\sigma}_{t}^{2}$ are 
TABLE 10. EGARCH(1,1) Model Asymmetry Tests

\begin{tabular}{llrl}
\hline & SBT & NSBT & PSBT \\
\hline DAX & 0.107 & 0.651 & -1.548 \\
Dow Jones & 0.357 & -0.429 & -1.765 \\
FTSE100 & 0.630 & 0.975 & -0.379 \\
MIB30 & 0.664 & 0.835 & -0.112 \\
\hline
\end{tabular}

Note: See Engle and $\mathrm{Ng}$ (1993) for the details regarding these tests.

independent from negative and positive $y_{t} / \hat{\sigma}_{t}$ shocks. As it is apparent in table 10, the EGARCH standardized residuals do not present any remaining asymmetry, showing that Nelson (1991) model is able to capture the leverage effect. Finally it can be deduced that the GARCH- $t$ model fits returns better than the standard GARCH, describing jointly the tail thickness of the unconditional distributions and the squared returns autocorrelations, while the EGARCH describes asymmetry but not unconditional kurtosis completely. Thus a fat-tailed EGARCH model is strongly recommended for the analyzed datasets.

\section{Estimation of SV models}

In this subsection, the standard SV with normal conditional distribution $(\mathrm{SV})$, the SV- $t$ with $t$ student conditional distribution (SV-t) and the asymmetric SV model (ASV) with normal conditional distribution are fitted. All models are estimated using the QML approach via the Kalman Filter suggested by Harvey et al. (1994) and Harvey and Shephard (1996).

Tables 11, 12 and 13 show the estimates and their standard deviations of the SV, SV-t and ASV models. It is important to note that estimates of the persistence parameter $\phi$ are very high in all series, even if significantly different from one (this means that volatility cannot be considered a random walk process). In particular, estimates of $\phi$ obtained from the application of SV and SV-t are very similar and are higher than the estimates obtained from ASV. This is mainly due to the greater number of parameters in the ASV model.

Referring to the asymmetric effect, the results obtained for the ASV model are very similar to those obtained for the EGARCH model. The 
TABLE 11. SV Model Estimates

\begin{tabular}{lccc}
\hline & $\phi$ & $\sigma_{\eta}$ & $\alpha$ \\
\hline \multirow{2}{*}{ DAX } & 0.989 & 0.115 & -0.013 \\
& $(0.003)$ & $(0.017)$ & $(0.000)$ \\
Dow Jones & 0.9942 & 0.0823 & -0.0569 \\
& $(0.0025)$ & $(0.0158)$ & $(0.000)$ \\
FTSE100 & 0.9930 & 0.0818 & -0.0661 \\
MIB30 & $(0.0025)$ & $(0.0131)$ & $(0.000)$ \\
& 0.971 & 0.153 & -0.037 \\
& $(0.006)$ & $(0.021)$ & $(0.000)$ \\
\hline
\end{tabular}

Note: Parentheses include the standard errors of the estimates.

TABLE 12. SV- $t$ Model Estimates

\begin{tabular}{lccccc}
\hline & $\phi$ & $\sigma_{\eta}$ & $\alpha$ & $v$ & $k \sim t$ \\
\hline \multirow{2}{*}{ DAX } & 0.990 & 0.112 & -0.093 & 37.58 & 3.881 \\
& $(0.003)$ & $(0.017)$ & $(0.033)$ & & \\
Dow Jones & 0.995 & 0.078 & -0.051 & 15.43 & 4.887 \\
& $(0.002)$ & $(0.016)$ & $(0.026)$ & & \\
FTSE100 & 0.993 & 0.076 & -0.007 & 18.88 & 3.294 \\
MIB30 & $(0.003)$ & $(0.014)$ & $(0.030)$ & & \\
& 0.973 & 0.146 & -0.235 & 13.60 & 3.771 \\
& $(0.006)$ & $(0.022)$ & $(0.064)$ & & \\
\hline
\end{tabular}

Note: Parentheses include the standard errors of the estimates.

parameter $\rho$, which represents the correlation between $\mathcal{E}_{t}$ and $\eta_{t}$ and hence the asymmetric effect, is negative in all series. Moreover, in its absolute value this coefficient is higher for Dow Jones and FTSE100. Table 13 reports kurtosis values for standardized returns after estimating the three models. SV- $t$ standardized returns have been transformed as in the GARCH- $t$ case. Consistent with the theoretical analysis outlined in section II, the standard model guarantees the best unconditional kurtosis fitting, so it is not necessary to resort to fat-tailed conditional distribution to replicate returns leptokurtic distribution. The SV asymmetric is the worst performer among the three models in describing outliers.

The Coefficient of Variation (CV) value, calculated as 
TABLE 13. Kurtosis Values for Adjusted Returns, SV, SV-t, and ASV Models

\begin{tabular}{lcccc}
\hline & $y_{\mathrm{t}}$ & SV-N & SV- $t$ & ASV \\
\hline DAX & 5.667 & 3.325 & 3.419 & 3.689 \\
Dow Jones & 8.058 & 2.872 & 3.840 & 4.449 \\
FTSE100 & 4.389 & 3.078 & 3.048 & 3.363 \\
MIB30 & 4.415 & 2.992 & 2.800 & 3.724 \\
\hline
\end{tabular}

TABLE 14. Coefficient of Variations for $S V, S V-t$ and ASV Models

\begin{tabular}{lccc}
\hline & SV-N & SV- $t$ & ASV-N \\
\hline DAX & 0.266 & 0.878 & 1.117 \\
Dow Jones & 1.013 & 0.840 & 0.925 \\
FTSE100 & 0.164 & 0.513 & 0.646 \\
MIB30 & 0.183 & 0.492 & 0.561 \\
\hline
\end{tabular}

$\exp \left(\sigma_{\eta}^{2} /\left(1-\phi^{2}\right)\right)$ is a measure of the relative strength of the level of conditional heteroskedasticity of returns series (Jacquier et al. [1994], Sandmann and Koopman [1998]). In particular, CV values around 1 indicate pronounced relative strength of the stochastic volatility process while values near to 0 signify that the model is close to constant volatility. Except in the case of the Dow Jones estimate, the standard SV coefficient of variation (see table 14) is too low to describe the heteroskedasticity shown by empirical returns. On the other hand, SVand SV asymmetric models present $\mathrm{CV}$ values which are more consistent with the strong heteroskedasticity of the data.

The LB test for autocorrelations in standardized prewhitened returns displays significant values for DAX returns, regardless of the model applied. This result is probably due to the presence of a residual linear autocorrelation in the standardized series. As for other datasets, the LB test does not reject the white noise hypothesis for the standardized residuals. Besides, the LB test shows the standard SV model failure in representing the second moment structure of returns, while $S V-t$ and $S V$ asymmetric specifications do not reject the IID hypothesis. For the MIB30 dataset none of the models can describe the strong squared returns autocorrelation (see table 15).

Finally, in table 16 results of the Engle and $\mathrm{Ng}$ (1993) diagnostics 
TABLE 15. LB Statistics for Standardized Residuals and Their Squared Values

\begin{tabular}{llll}
\hline & SV-N & SV- $t$ & ASV-N \\
\hline$L B(30)$ & & & \\
DAX & 44.218 & 43.360 & 44.464 \\
Dow Jones & 39.317 & 37.358 & 38.463 \\
FTSE100 & 25.288 & 23.484 & 24.300 \\
MIB30 & 33.059 & 25.724 & 25.668 \\
$L B^{2}(30)$ & & & \\
DAX & 58.759 & 24.422 & 29.534 \\
Dow Jones & 24.050 & 35.924 & 19.595 \\
FTSE100 & 26.590 & 28.444 & 30.368 \\
MIB30 & 69.928 & 76.036 & 63.999 \\
\hline
\end{tabular}

Note: $L B$ is for statistics on standardized residuals and $L B^{2}$ is for their squared values. The critical value for the above LB statistics is $\chi^{2}(28)=41.337$.

TABLE 16. ASV-N Model Asymmetry Tests

\begin{tabular}{lrrr}
\hline & SBT & NSBT & PSBT \\
\hline DAX & -0.601 & 0.628 & -2.731 \\
Dow Jones & -1.172 & -2.919 & -2.694 \\
FTSE100 & -0.159 & 0.459 & -1.161 \\
MIB30 & 0.319 & -1.932 & 1.897 \\
\hline
\end{tabular}

Note: See Engle and $\mathrm{Ng}$ (1993) for the details regarding these tests.

tests are presented for the SV asymmetric model. Although first proposed and applied to GARCH asymmetric models, the three tests can also be applied to volatility models which are not members of the GARCH family (Engle and $\mathrm{Ng}$ [1993]). Results show a good performance for the asymmetric model in catching the FTSE100 and MIB30 returns asymmetry, and partial and total inadequacy in capturing the same, strongly present effect for DAX and Dow Jones returns. To summarize, diagnostic tests show that the standard version of the SV model is adequate to explain unconditional kurtosis of the datasets analyzed. For more leptocurtic returns distributions, as it is often encountered in literature (see, e.g., Taylor (1986), Bollerslev et al. (1994), Harvey et al. (1994), Shephard (1996), Drobetz and Zimmermann (2003), Engle and Patton (2001) and Peters (2001) among others), probably a SV- $t$ or more fat-tailed model would be a better choice. On the other hand, the standard SV model cannot adequately 
TABLE 17. Kurtosis Values for Standardized Residuals of All Models

\begin{tabular}{lcccc}
\hline Model & DAX & Dow Jones & FTSE100 & MIB30 \\
\hline GARCH(1,1) & 4.118 & 4.707 & 3.514 & 3.578 \\
SV-N & 3.325 & 2.872 & 3.078 & 2.992 \\
GARCH(1,1)- $t$ & 3.065 & 3.018 & 3.011 & 3.003 \\
SV- $t$ & 3.419 & 3.840 & 3.048 & 2.800 \\
EGARCH(1,1) & 4.064 & 4.466 & 3.651 & 3.653 \\
ASV-N & 3.689 & 4.449 & 3.363 & 3.724 \\
\hline
\end{tabular}

describe conditional heteroskedasticy and squared returns autocorrelations, while the SV-t, although it performs slightly worse than the standard formulation in explaining the unconditional kurtosis, does not reveal any shortcoming in describing heteroskedasticity and second moment autocorrelations. The only exception is the MIB30 index which squared returns autocorrelations could not be described by any model. Finally, the asymmetric model has difficulty in capturing unconditional kurtosis, especially the asymmetric effect when it is strong (as in the DAX and MIB30 case).

\section{Comparing performances}

First of all, the models' adequacy to describe the typical thick-tails feature are compared. In particular, the kurtosis values of the standardized returns $y_{t} / \sigma_{\mathrm{t}}$ through the volatilities estimated by the application of each model are considered. The standardized returns should be approximately distributed as Gaussian white noises. The more the estimated kurtosis approaches the Gaussian kurtosis value of 3, the more the model can be considered adequate to capture the excessive number of outliers typical of unconditional distributions returns. Comparing the two standard formulations, the SV model shows values of kurtosis closer to the Gaussian kurtosis value of 3 than the GARCH one, indicating a better goodness of fit in representing fat tails (table 17).

These results confirm those obtained by other authors, such as Geweke (1995), Shephard (1996), Kim et al. (1998), who compared goodness of fit between GARCH and SV models using different methods. Asymmetric SV is slightly better than EGARCH in describing fat tails, even though it is not as good as the standard model. Finally, it is important to note that GARCH- $t$ model is not only better able to 
TABLE 18. Mean Absolute Deviations Between Induced ACFs and Squared Returns ACFs

\begin{tabular}{lcccc}
\hline Model & DAX & Dow Jones & FTSE100 & MIB30 \\
\hline GARCH(1,1) & 0.172 & 0.285 & 0.287 & 0.102 \\
SV-N & 0.036 & 0.058 & 0.080 & 0.044 \\
GARCH(1,1)- $t$ & 0.154 & 0.142 & 0.217 & 0.148 \\
SV- $t$ & 0.042 & 0.093 & 0.026 & 0.060 \\
EGARCH(1,1) & 0.079 & 0.099 & 0.089 & 0.070 \\
ASV-N & 0.011 & 0.005 & 0.018 & 0.008 \\
\hline
\end{tabular}

capture empirical leptocurtosis (fat-tails) than the SV-t model, but is also the best in relation to the other models.

In order to compare the fit of the models in describing autocorrelations shown by squared returns, the autocorrelation function (ACF) and induced ACF are calculated by applying the theoretical equations provided in literature (Bollerslev [1986], Taylor [1994], He et al. [1999]), using parameter estimates and on the basis of a minimum distance criterion (mean absolute deviations), the best-fitting model is established.

Results reported in table 18 show that SV models dominate GARCH in describing squared empirical ACFs. Likewise, among the different specifications considered here, asymmetric models guarantee, for each class of models, the best goodness of fit regarding the specific returns feature analyzed. Besides, heavy tailed distributions perform better than standard formulations, and overall it is important to highlight that the best GARCH-type model (EGARCH) under-performs even the worst $\mathrm{SV}$ model $\left(S V_{-t}\right)$. Furthermore, see figure 1 of the ACFs built from the parameters estimates of EGARCH (short dash line) and asymmetric SV (long dash line) compared with sample squared returns autocorrelations (dots) for the four indexes analyzed.

Tables 10 and 16 provide results of Engle and $\mathrm{Ng}$ (1993) tests applied to standardized residuals from EGARCH and SV asymmetric models. As shown by the reported values, EGARCH captures the leverage effect better than the SV asymmetric model. Indeed, while for FTSE100 and MIB30 indexes both models ensure the same fitting, for the DAX and Dow Jones indexes, i.e., the two more asymmetric datasets, tests show large values for the SV model, while for EGARCH no significant values are presented. 

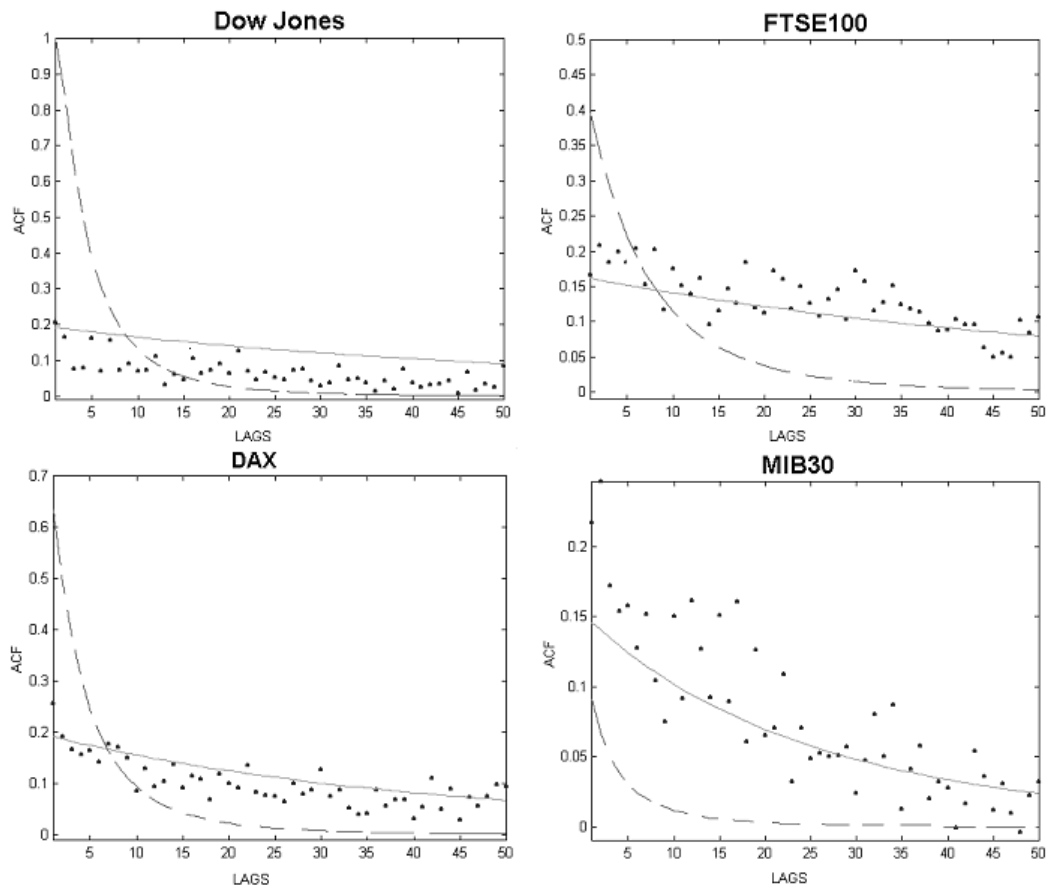

Figure 1.-ACFs Based on EGARCH (dashed line) and Asymmetric SV Model (line) vs. the Empirical Squared Autocorrelations (dots).

\section{Conclusion}

On the basis of the results which emerge while using four different data sets of financial returns, it is possible to conclude that the Stochastic Volatility model, in its standard formulation, provides a better goodness of fit than the standard GARCH one. In particular, the normal SV dominates the normal GARCH both in describing heavy tailed unconditional returns and in representing the strong and slow decaying squared returns sample autocorrelations. As regards other more complex formulations, the outcomes are not so clearly in favour of SV models: GARCH-t formulation fits outliers better than SV-t, while it proves not to be as effective as $\mathrm{SV}$ in explaining squared autocorrelations.

Finally, asymmetric models, i.e., EGARCH and SV asymmetric, are not as good as the previous ones in describing fat tails, but are very 
suitable in approximating squared returns ACFs. Asymmetric SV is superior in capturing heavy tails and autocorrelations, while EGARCH is better in describing the asymmetric effect when this is particularly strong.

These results provide a preliminary analysis for the choice of the best model to forecast the volatility of financial returns. In fact, when the return distribution is characterized by fat-tails (or asymmetry) the GARCH-t (or EGARCH) models are recommended. Otherwise, when return distribution is normal, the SV model is more adequate.

\section{References}

Abramovitz, M. and N. C. Stegun, 1970. Handbook of mathematical functions, New York: Dover Publications.

Akaike H., 1974, A new look at statistical model identification. IEEE Transactions on Automatic Control, 19: 716-723.

Andersen T.G., 1994. Stochastic autoregressive volatility: A framework for volatility modeling. Mathematical Finance, 4: 75-102.

Andersen T. G., H. Chung and B.E. Sørensen, 1999. Efficient method of moments estimation of a stochastic volatility model: A monte Carlo study. Journal of Econometrics, 91: 61-87.

Andersen, T.G. and B. E. Sørensen, (1996). GMM estimation of a stochastic volatility model: A monte Carlo study. Journal of Business and Economic Statistics, 14(3): 328-352.

Bai, X., J. R. Russell and G. C. Tiao, 2001. Kurtosis of GARCH and stochastic volatility models with non-normal innovations. Working Paper. Chicago: University of Chicago.

Bali, T. G., 2000. Testing the empirical performance of stochastic volatility models of the short-term interest rate. Journal of Financial and Quantitative Analysis, 35(2): 191-215.

Bollerslev T., 1986. Generalized autoregressive conditional heteroskedasticity. Journal of Econometrics, 31: 307-327.

Bollerslev T., 1987. A conditional heteroskedastic time series model for speculative prices and rates of return. Review of Economics and Statistics, 69: 542-547.

Bollerslev T., R. F. Engle and R. F. Nelson, 1994. ARCH Models. In R.F. Engle and D.L. McFadden (eds). Handbook of Econometrics, ch. 49, vol. IV. Elsevier.

Box G.E.P. and G.M. Jenkins, 1976. Time Series Analysis, Forecasting and Control. San Francisco: Holden Day.

Campbell J.Y. and L. Hentschel, 1992. No news is good news: An asymmetric model of changing volatility in stock returns. Journal of Financial Economics, 31: 281-318. 
Campbell J.Y., A.W. Lo and A.C. MacKinlay, 1997. The Econometrics of Financial Markets. Princeton: Princeton University Press.

Chib S., F. Nardari and N. Shephard, 2001. Markov chain monte carlo methods for stochastic volatility models. Journal of Econometrics, forthcoming.

Clark P. K., 1973. A subordinated stochastic process model with finite variance fo speculative prices. Econometrica, 41(1), 135-159.

Danielsson J. and J.F. Richard, 1993. Accelerated Gaussian importance sampler with application to dynamic latent variable models. Journal of Applied Econometrics, 8: 153-173.

Drobetz W. and H. Zimmermann, 2003. Anatomy of volatility and stock market correlations. In Zimmermann H., Drobetz W., and Oertmann P. (Eds). Global Asset Allocation, ch. 3.

Durbin J. and S.J. Koopman, 1997. Monte Carlo maximum likelihood estimation for non-Gaussian state space models. Biometrika, 84: 669-684.

Engle R.F., 1982. Autoregressive conditional heteroskedasticity with estimates of the variance of U.K. inflation. Econometrica, 50: 987-1008.

Engle R.F. and V. Ng, 1993. Measuring and testing the impact of news on volatility. Journal of Finance, 48: 1749-1778.

Engle R.F. and A.J. Patton, 2001. What good is a volatility model? Quantitative Finance, 1(2): 237-245.

Gallant A.R., D.A. Hsieh and G. Tauchen, 1997. Estimation of stochastic volatility models with diagnostics. Journal of Econometrics, 81: 159-192.

Gallant A.R. and G.E. Tauchen, 1996. Which moments to match? Econometric Theory, 12: 657-681.

Geweke J., 1994. Comment on Bayesian analysis of stochastic volatility. Journal of Business and Economic Statistics, 12(4): 397-399.

Geweke J., 1995. Bayesian comparison of econometric models. Working Paper: Federal Reserve Bank of Minneapolis.

Ghysels E., A. Harvey and E. Renault, 1996. Stochastic volatility. Monteal: Scientific Series, CIRANO.

Gouriéroux C., A. Monfort and E. Renault, 1993. Indirect inference. Journal of Applied Econometrics, 8: 85-118.

Harvey A., E. Ruiz and N. Shephard, 1994. Multivariate stochastic variance models. Review of Economic Studies, 61: 247-264.

Harvey A. and N. Shephard, 1993. Estimation and testing of stochastic variance models. Discussion Paper: STICERD, London School of Economics and Political Science.

Harvey A. and N. Shephard, 1996. Estimation of an asymmetric volatility model for asset returns. Journal of Business and Economic Statistics, 14(4): 429-434.

He C., Terasvirta T. and H. Malmsten, 1999. Fourth moments structure of a family of first-order exponential GARCH models. Working Paper: Stockholm School of Economics.

Jacquier E., N.G. Polson and P.E. Rossi, 1994. Bayesian analysis of stochastic 
volatility models. Journal of Business and Economic Statistics, 12(4): 371-417.

Jacquier E., N.G. Polson and P.E. Rossi, 2001. Bayesian analysis of a stochastic volatility model with leverage effect and fat tails. Journal of Econometrics, forthcoming.

Jarque C.M. and A.K. Bera, 1987. Efficient tests for normality, homoscedasticity and serial independence for regression residuals. Economics Letters, 6: 255-259.

Kim S., N. Shephard and S. Chib, 1998. Stochastic volatility: likelihood inference and comparison with ARCH models. Review of Economic Studies, 65: 361-394.

Lamourex C.G. and W.D. Lastrapes, 1994. Endogenous trading volume and momentum in stock-return volatility. Journal of Business and Economic Statistics, 12(2): 253-260.

Ljung G.M. and G.E.P. Box, 1978. On a measure of lack of fit in time series models. Biometrika, 65: 553-564.

Liesenfeld R. and R.C. Jung, 2000. Stochastic volatility models: Conditional normality versus heavy-tailed distributions. Journal of Applied Econometrics, 15(2): 137-160.

Lundberg S. and T. Terasvirta, 2001. Evaluating GARCH models. Journal of Financial Econometrics, forthcoming.

Mikosch T. and C. Starica, 2000. Limit theory for the sample autocorrelations and extremes of a GARCH(1,1) process. Annuals of Statistics, 28: 885-908.

Nelson D.B., 1991. Conditional heteroskedasticity in asset returns: A new approach. Econometrica, 59: 347-370.

Nicolis O., Aprile 2000. Stima non parametrica della media e varianza condizionale con i polinomi locali. Società Italiana di Statistica, XL Riunione Scientifica, Firenze 26-28.

Peters J., 2001. Estimating and forecasting volatility of stock indices using asymmetric GARCH models and, skewed student-t densities. Working Paper: Ecole d'Administration des Affaires, University of Liege, Belgium.

Ruiz E., 1994. Quasi-maximum likelihood estimation of stochastic volatility models. Journal of Econometrics, 64: 289-306.

Sandmann G. and S.J. Koopman, 1998. Estimation of stochastic volatility models via monte Carlo maximum likelihood. Journal of Econometrics, 87: 271-301.

Schwert, G. W., 1989. Why does stock market volatility change over time? Journal of Finance, XLIV(5) 1115-1153.

Shephard N., 1996. Statistical aspects of ARCH and stochastic volatility. In Cox D.R, Hinkley D.V. and Barndorff-Nielsen O.E.(eds). Time Series Models, ch. 1. Chapman and Hall.

Starica C. and O. Pictet, 1997. The tales the tails of GARCH(1,1) Processes tell. Working Paper: Departments of Mathematics, Chalmers School of Business, Ghoteborg, Sweden. 
Tauchen G. E. and M. Pitts, 1983. The price variability relationship on speculative markets. Econometrica, 51(2): 485-505.

Tauchen G.E., H. Zhang and M. Liu, 1996. Volume, volatility, and leverage: A dynamic analysis. Journal of Econometrics, 74: 177-208.

Taylor S., 1986. Modelling financial time series. New York: Wiley.

Taylor S., 1994. Modeling Stochastic volatility: A Review and comparative study. Mathematical Finance, 4(2): 183-204.

Terasvirta T., 1996. Two stylized facts and the GARCH(1,1) model. Working Paper: Stockholm School of Economics. 